\title{
The Fit Accuracy of Removable Partial Denture Metal Frameworks Using Conventional and 3D Printed Techniques: An In Vitro Study
}

\author{
Salwa O Bajunaid ${ }^{1}$, Bashaer Altwaim ${ }^{2}$, Muneera Alhassan ${ }^{3}$, Rawan Alammari ${ }^{4}$
}

\begin{abstract}
Aim: To evaluate the accuracy of removable partial denture (RPD) metal frameworks fabricated by the conventional lost-wax (CLW) technique and those made by the selective laser melting (SLM).

Materials and methods: A dentoform of a mandibular Kennedy class III, modification 1 dental arch were surveyed, and rest seats were prepared on the abutment teeth. The dentoform was then duplicated into a metal die which was used as a reference model. Thirty RPD metal frameworks were fabricated by two techniques; fifteen for each technique. Polyvinyl siloxane (PVS) impression material was painted on the intaglio surface of the rests of each framework which is then seated on the reference die. PVS specimens that represent the gap under the rest were measured in four zones: buccal, lingual, marginal and central by a single examiner using a digital microscope at 50x in micrometers.

Results: Comparison between the two techniques for each abutment tooth revealed that the CLW technique had better fit in one tooth, while the SLM technique showed a better fit in two teeth.

Regarding the edentulous span length within the SLM technique, the long edentulous span had a significantly better fit. When comparing the four measured rest zones, it was found that in the CLW technique group, the marginal zone had the highest fit accuracy while the lingual zone showed the lowest fit accuracy. In the SLM group, the central zone had the best fit and the buccal zone had the worst fit.
\end{abstract}

Conclusion: RPD frameworks fabricated using the SLM technique showed better fit accuracy than those made by the CLW technique, however, the difference was not statistically significant.

Clinical significance: SLM is a promising technique for the fabrication of RPD frameworks in routine clinical practice.

Keywords: Fit accuracy, Laboratory research, Lost-wax technique, Removable partial denture framework, Selective laser melting laboratory research.

The Journal of Contemporary Dental Practice (2019): 10.5005/jp-journals-10024-2542

\section{INTRODUCTION}

$\mathrm{N}$ owadays, the improvement in oral health maintenance resulted in a fewer number of missing teeth which led to a greater need to treat partially edentulous as compared to completely edentulous patients. $^{1,2}$

Replacement of the missing teeth and their associated structures is essential to restore masticatory function, satisfy esthetics and phonetics, and to prevent unwanted movement of the opposing or the adjacent teeth (supra eruption/drifting). ${ }^{3}$

Some clinical situations would necessitate the use of a RPD or prosthesis. These include but not limited to cases of long edentulous spans, lost or severely resorbed residual ridges and absence of posterior abutments. Moreover, some patients are not willing to undergo surgery for the placement of endosseous implants, while others do not want their sound teeth to be prepared as abutments for a fixed partial prosthesis.

Additionally, some people have financial limitations and are unable to afford more expensive treatment options. ${ }^{3}$ In such cases, a removable partial prosthesis is a cost-effective treatment modality and despite the advancement in the other approaches for tooth replacement, it will still be an important treatment option., ${ }^{1,4}$

For more than 70 years, the primary method of RPD metal framework fabrication was the CLW technique. ${ }^{5}$ However, this technique involves many laboratory procedures which are susceptible to accumulative human errors. ${ }^{6}$ Therefore, a significant need exists to evaluate new RPD framework materials, design and fabrication technologies, ${ }^{7}$ and many patients will require

\begin{abstract}
${ }^{1}$ Department of Prosthetic Dental Sciences, College of Dentistry, King Saud University, Riyadh, Kingdom of Saudi Arabia

${ }^{2-4}$ College of Dentistry, King Saud University, Riyadh, Kingdom of Saudi Arabia
\end{abstract}

Corresponding Author: Salwa O Bajunaid, Department of Prosthetic Dental Sciences, College of Dentistry, King Saud University, Riyadh, Kingdom of Saudi Arabia, Phone: +(966)590028784, e-mail:dr_prosth@ hotmail.com

How to cite this article: Bajunaid SO, Altwaim B, Alhassan M, Alammari R. The Fit Accuracy of Removable Partial Denture Metal Frameworks Using Conventional and 3D Printed Techniques: An In Vitro Study. J Contemp Dent Pract 2019;20(4):476-481.

Source of support: Nil

Conflict of interest: None

replacement of missing teeth. Although current treatment options also include fixed partial dentures and implants, RPDs At the beginning of the1970s, there was an evolution in digital dentistry through the development of computer-aided design and computer-aided manufacturing (CAD/CAM) technology. Until the early 1980s, subtractive manufacturing was the basic assembly method. ${ }^{5}$ Recently, additive manufacturing was introduced to the dental field with various techniques. These include selective laser sintering (SLS) for non-metallic materials (i.e., ceramic or polymers) and selective laser melting (SLM) technique for metallic alloys. ${ }^{5,8}$

SLM is a material-addition technique in which the successive layers are created using a high energy laser beam that selectively

(c) The Author(s). 2019Open Access This article is distributed under the terms of the Creative Commons Attribution 4.0 International License (https://creativecommons. org/licenses/by-nc/4.0/), which permits unrestricted use, distribution, and non-commercial reproduction in any medium, provided you give appropriate credit to the original author(s) and the source, provide a link to the Creative Commons license, and indicate if changes were made. The Creative Commons Public Domain Dedication waiver (http://creativecommons.org/publicdomain/zero/1.0/) applies to the data made available in this article, unless otherwise stated. 
fuses and consolidates metal powder to generate a complex threedimensional object. ${ }^{9,10}$ Its key advantages are the ability to directly produce high-precision metal parts in their full density. Also, the remaining unprocessed metal powder can be recycled so there will be no material waste. In addition, this technique consumes less time and is more economical in designing and fabrication. ${ }^{9-11}$ Hence, SLM can be a promising technique in the denta I field.

Fitting of a removable partial denture RPD framework is one of the most important requirements for the success of the prosthesis. Any misfit might cause discomfort which could refrain many patients from wearing their prosthesis. Also, the improper fit may result in the movement of the associated teeth. ${ }^{3}$

To the knowledge of the authors, a literature search revealed no published experimental or clinical trials that compared the fit of RPD frameworks constructed by the conventional lost-wax CLW technique with those produced by the SLM technology.

Therefore, the aim of this study was to compare the fit accuracy of the conventional lost-wax CLW technique RPD frameworks with selective laser melted ones. The null hypothesis is that there is no significant difference in the fit: (a) between the CLW and the SLM frameworks, (b) between the long and the short edentulous spans within each technique and (c) between the four zones within the rest (buccal, lingual, marginal and central) for each technique.

\section{Materials and methods}

This study was conducted at the College of Dentistry at King Saud University, Riyadh, Kingdom of Saudi Arabia. Thirty removable partial denture frameworks were fabricated, constituting two groups with fifteen frameworks for each group. The frameworks in the first group (control group) were fabricated using the CLW technique and the second group (test group) contained frameworks that were made by the SLM technique. A mandibular dentoform with the first molar tooth missing on the left side and the first molar and second premolar teeth missing on the right side of the arch was used. The dentoform was surveyed to determine a common path of insertion and to find undercut areas on the abutment teeth; this was followed by tripoding of the model.

Rest seats were prepared on the primary abutment teeth as follows: a mesial occlusal rest seat on the second molar (\#37) and a distal occlusal rest seat on the second premolar (\#35) in the left side. On the right side, a mesial occlusal rest seat on the second molar (\#47) and a distal occlusal rest seat on the first premolar (\#44)as shown in

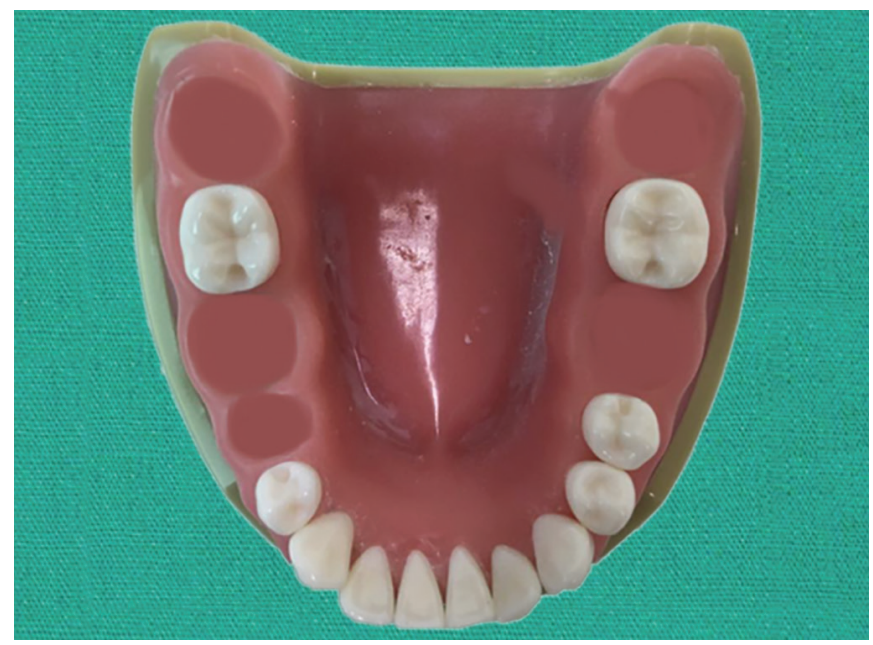

Fig. 1: Primary abutment teeth with rest seats preparation
Figure 1. All the teeth were numbered according to the Federation Dentaire International (FDI) teeth numbering system. The denotform was then duplicated into a cobalt-chromium die which was fabricated by the SLM technique. This metal model was used as a reference to evaluate the fit of all RPD frameworks as shown in Figure 2.

In the first group, fifteen metal frameworks were fabricated by following the steps of the CLW technique guided by the manufacturers' instructions. The metal die was duplicated using irreversible silicone duplicating material (Deguform ${ }^{\circledast}$ Plus, DeguDent, Hanau, Germany) to obtain the mold. Investment material (Wirovest and BegoSol, BEGO, Lincoln, United States) was poured into this mold to produce fifteen refractory casts. Casting wax (Model casting wax, YETI Dentalprodukte, Engen, Germany) was adapted to the refractory casts according to the selected framework design. After that the casts were invested (Wirovest and BegoSol, BEGO, Bremen, Germany) and Cobalt-chromium alloy (Solidur $\mathrm{CoCr}$, YETI Dentalprodukte, Engen, Germany) was used to fabricate the RPD frameworks.

Finishing off all frameworks was conducted by sandblasting, tungsten carbide burs and mounted stone burs. For the polishing procedure electrolyte machine (Eltropol 300, BEGO, Lincoln, United States) and silicone rubber burs were used.

In the second group, the frameworks were fabricated using SLM technology. The metal die was scanned with a fully automated optical structured-light Scanner S600 ARTI (Zirkonzhan, South Tyrol, Italy). Then surveying and designing of the removable partial denture frameworks were built virtually in a standard tessellation language file (STL) in a 3D format (3 Shape dental software systems, Copenhagen, Denmark)as shown in Figure 3. The STL file was then transferred to the rapid prototyping machine (Mlap Cusing Machine fiber laser100 $\mathrm{W}_{(} \mathrm{cW}_{\mathrm{f}}$, Concept Laser, Germany) and the definitive frameworks were produced using cobalt-chromium alloy powder (Remanium star CL cobalt-chrome alloy, Dentaurum, Karlsruhe, Germany)as shown in Figure 4. The frameworks were finished by sandblasting and diamond burs then polished by rubber points.

To evaluate the fit of the frameworks, light body polyvinyl siloxane impression material (PVS) (Express ${ }^{\mathrm{TM}}$, 3M ESPE, Minnesota, USA) was painted on the intaglio surfaces of the rests. ${ }^{12}$ The RPD framework was held in place on the metal die with finger pressure that was maintained through the sitting time determined by the manufacturer as shown in Figure 5. The accuracy of the fit was evaluated by measuring the thickness of the PVS material which

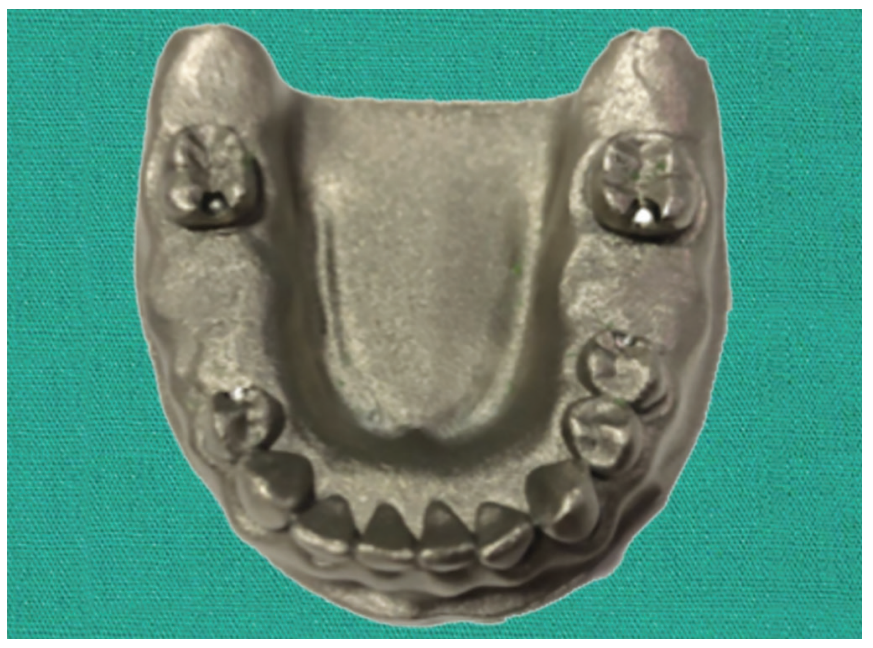

Fig. 2: A cobalt-chromium model used to evaluate the fit of all RPD frameworks 


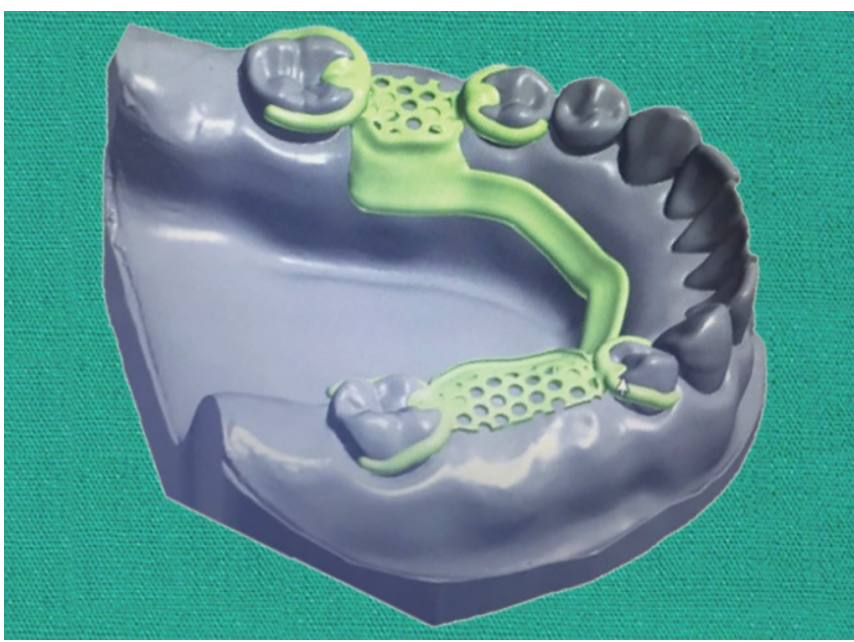

Fig. 3: Virtual surveying and designing of the RPD frameworks

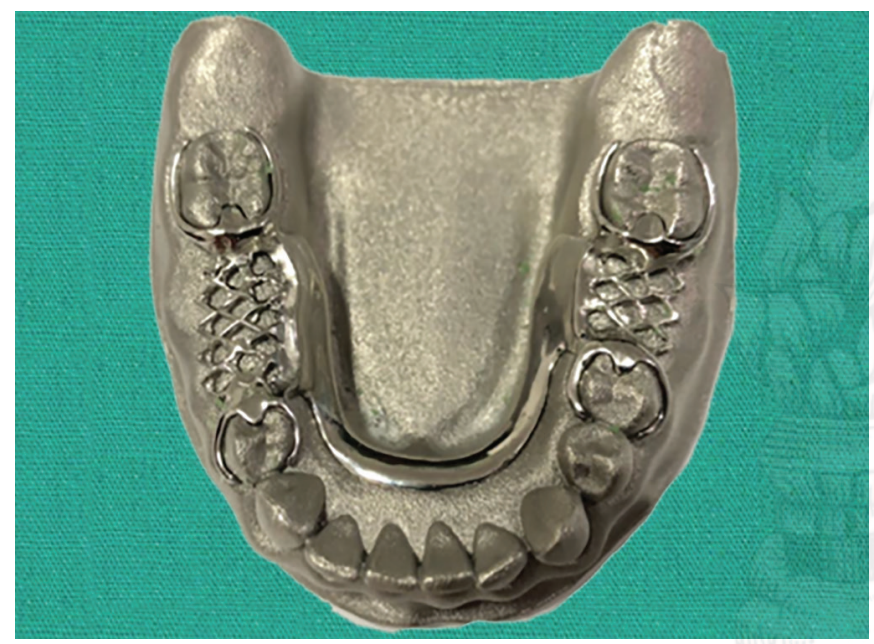

Figs 5A and B: (A) RPD framework fabricated by CLW technique seated on the reference model; (B) RPD framework fabricated by SLM technique seated on the reference model

represents the gap between the rest and its corresponding rest seat area. The measurements were calculated in micrometer using a Digital Microscope (KH-7700, Hirox, Tokyo, Japan) at a magnification power of 50x as shown in Figure 6. The impression specimen for each rest was divided into four zones: buccal, lingual, marginal and central. ${ }^{13}$

All measurements on the digital microscope were obtained by a single examiner. The intraexaminer reliability was calibrated by Cronbach's alpha test at 0.998 . The intraclass correlation coefficient was 0.996 which indicates high measuring accuracy.

\section{Results}

A total of 120 PVS specimens, each with four zones (total 480 measurements) represented the gap between the metal frameworks' rests and their corresponding rest seats. The specimens were obtained equally from the CLW and the SLM groups.

Statistical analysis was performed using statistical software (IBM SPSS Statistics V22.0, United States). The significance threshold was set at 0.05 .

For each tooth, the means of the gap distance measured in micrometers for both the CLW and the SLM groups were compared

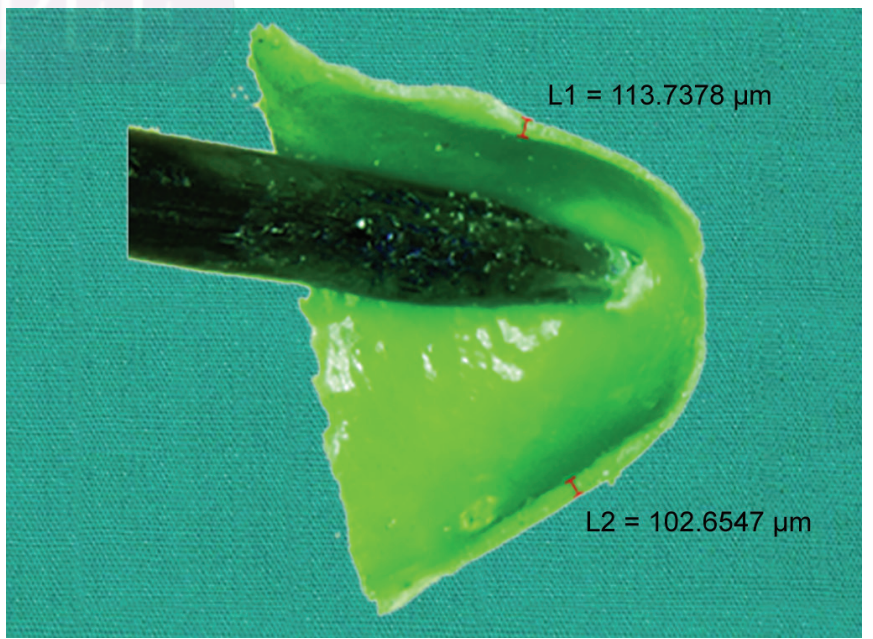

Fig. 6: The measurements calculated in micrometer using a digital microscope at magnification power of $50 x$

using independent t-test.

The mean of the total gap's measurements of the four zones 
(buccal, lingual, marginal and central) in each tooth was calculated in both the CLW and SLM groups. The results showed that, for tooth \#37, there was no significant difference in the gap between the metal frameworks' rests and their corresponding rest seats between the CLW and SLM groups: $243.56 \pm 166.34 \mu \mathrm{m}$ and 265.62 $\pm 171.34 \mu \mathrm{m}$, respectively $(p>0.05)$. However, teeth \#35 and \#47 showed a significantly better fit in the SLM group (251.51 \pm 130.51 $\mu \mathrm{m})$ and $(211.30 \pm 140.89 \mu \mathrm{m})$; respectively versus CLW group $(313.58 \pm 175.13 \mu \mathrm{m})$ and $(286.13 \pm 195.49 \mu \mathrm{m})$, respectively $(p<$ 0.05). Tooth \#44 had a significantly better fit in the CLW group $(275.18 \pm 158.84 \mu \mathrm{m})$ versus SLM group $(360.20 \pm 208.91 \mu \mathrm{m})(p$ $<0.05$ ) (Table 1).

Each framework was designed to have a short span edentulous area on the left side and a long span edentulous area on the right side. When comparing the contralateral abutment teeth within each group using the independent T-test, the measurements revealed no significant difference between short edentulous spans(278.58 \pm $173.67 \mu \mathrm{m})$ and long edentulous spans $(280.65 \pm 177.45 \mu \mathrm{m})$ within the CLW technique $(p>0.05)$. However, the SLM technique had a significantly better fit in the long edentulous spans $(285.76 \pm 192.54$ $\mu \mathrm{m})$ compared to short edentulous spans $(258.57 \pm 151.83 \mu \mathrm{m})$ $(p<0.05)$ (Table 2).

Within each group, all measured zones (buccal, lingual, marginal and central) for every single rest were compared using the one-way
ANOVA and post hoc Tukey's test. The results revealed that the best-fitted zone in the CLW technique group was the marginal zone $(172.71 \mu \mathrm{m})$, followed by the central zone $(195.40 \mu \mathrm{m})$ and the buccal zone $(237.29 \mu \mathrm{m})$. While the lingual zone showed the worst fit $(513.06 \mu \mathrm{m})$, with significantly increased gap distance (Table 3A).

Regarding the SLM technique group, the central zone (155.31 $\mu \mathrm{m})$ had the best fit, followed by the marginal zone $(165.04 \mu \mathrm{m})$ and the lingual zone $(347.16 \mu \mathrm{m})$. The highest thickness of the PVS impression material was found in the buccal zone $(421.14 \mu \mathrm{m})$ (Table 3B).

When the overall means of CLW group $(279.61 \pm 175.21 \mu \mathrm{m})$ and the SLM group $(272.16 \pm 173.55 \mu \mathrm{m})$ were compared using the independent T-test, the difference was not significant $(p>0.05)$ (Table 4).

\section{Discussion}

Even with the advancement and diversity of treatment modalities provided for the partially edentulous patients, the removable partial denture is still a widely used treatment option in many clinical cases. Nowadays, there is a global trend towards utilizing digitally fabricated dental prostheses via rapid prototyping; by either additive or subtractive techniques. ${ }^{5,14}$ Additive techniques have been recently used in RPD fabrication. ${ }^{10,15,16}$ and the selective laser melting technique (SLM) is one of the promising techniques

Table 1: Means and standard deviations of the gaps between the rests and their rest seats for CLW and the SLM groups for each individual tooth in micrometers $(\mu \mathrm{m})$

\begin{tabular}{|c|c|c|c|c|c|}
\hline Tooth \# & $N$ & Technique & Mean & Std. deviation & $p$ value \\
\hline \multirow{2}{*}{ \#37 } & 60 & CLW & 243.56 & 166.34 & \multirow{2}{*}{0.882} \\
\hline & 60 & SLM & 265.62 & 171.35 & \\
\hline \multirow{2}{*}{ \#35 } & 60 & CLW & 313.59 & 175.13 & \multirow{2}{*}{0.041} \\
\hline & 60 & SLM & 251.51 & 130.52 & \\
\hline \multirow{2}{*}{$\# 44$} & 60 & CLW & 275.18 & 158.85 & \multirow{2}{*}{0.020} \\
\hline & 60 & SLM & 360.21 & 208.91 & \\
\hline \multirow{2}{*}{$\# 47$} & 60 & CLW & 286.13 & 195.49 & \multirow{2}{*}{0.007} \\
\hline & 60 & SLM & 211.31 & 140.89 & \\
\hline
\end{tabular}

Table 2: Means and standard deviations of the gaps between the rests and their rest seats for CLW and the SLM groups for long and short spans within the same technique in micrometers $(\mu \mathrm{m})$.

\begin{tabular}{llllll}
\hline Technique & $N$ & Span length & Mean & Standard deviations & $p$ value \\
\hline \multirow{2}{*}{ CLW } & 120 & Short & 259.37 & 162.73 & 185.32 \\
\\
\multirow{2}{*}{ SLM } & 120 & Long & 299.86 & 196.09 & 0.07 \\
& 120 & Short & 312.92 & 136.73 & 0.00 \\
\hline
\end{tabular}

Table 3A: Means and standard deviations of the gaps between the rests and their rest seats for the CLW group for each zone in micrometers $(\mu \mathrm{m})$

\begin{tabular}{lllll}
\hline & & \multicolumn{3}{c}{ Subset for alpha $=0.05$} \\
\cline { 3 - 5 } Zone & $N$ & 1 & 2 & 3 \\
\hline Marginal & 60 & 172.71 & - & - \\
Central & 60 & 195.40 & 195.40 & - \\
Buccal & 60 & - & 237.29 & - \\
Lingual & 60 & - & - & 513.06 \\
$p$ value & - & 0.670 & 0.160 & 1.000 \\
\hline
\end{tabular}

Table 3B: Means and standard deviation of the gaps between the rests and their rest seats for the SLM group for each zone in micrometers $(\mu \mathrm{m})$.

\begin{tabular}{llccc}
\hline & & \multicolumn{3}{c}{ Subset for alpha $=0.05$} \\
\cline { 3 - 5 } Zone & $N$ & 1 & 2 & 3 \\
\hline Central & 60 & 155.31 & & \\
Marginal & 60 & 165.04 & & \\
Lingual & 60 & & 347.16 & 421.14 \\
Buccal & 60 & & & 1.000 \\
$p$ value & & 0.977 & 1.000 & \\
\hline
\end{tabular}


Table 4: Overall means and standard deviations of the gaps between the rests and their rest seats for the CLW and the SLM groups in micrometers $(\mu \mathrm{m})$.

\begin{tabular}{lllll}
\hline Technique & $N$ & Mean & Std. Deviation & $p$ \\
\hline CLW & 240 & 279.61 & 175.21 & 0.678 \\
SLM & 240 & 272.16 & 173.55 & \\
\hline
\end{tabular}

in digital dentistry. ${ }^{17}$ Many studies evaluated different parameters in selective laser melted cobalt chromium RPD frameworks. These parameters include rigidity and density, the strength of RPD clasp and patient satisfaction.

Those studies concluded that overall SLM had satisfactory results, ${ }^{10,18,19}$ based on a wax model (BEGO USA. In the present study, one additional important parameter was measured, the fit accuracy of the rests of the removable partial denture frameworks fabricated by the conventional lost-wax technique (CLW) or by the selective laser melting technology (SLM).

The null hypothesis tested in this research project could not be rejected except for the comparison of the four zones of the rests within each technique group and the comparison between the two edentulous spans within the SLM technique group.

When the gap measurement of all four teeth was compared between the two techniques, it was found that two teeth (\#35 and \#47) had a significantly better fit in the SLM technique while only one tooth had a significantly better fit in the CLW technique (\#44) (Table 1).

Regarding the length of the edentulous span, the present study showed that the long edentulous span had a significantly better fit in the SLM technique group. On the other hand, there was no significant difference in the span length within the CLW technique group (Table 2). This is in contrast to the results of two previous studies which compared the adaptation of conventionally fabricated RPD frameworks to short versus long edentulous spans. Akee ${ }^{20}$ found that the long span RPD had a better fit in comparison to the short span while Anan et al. ${ }^{21}$ found that the short span had a better fit.

In the present study, the best well-fitted zone in the CLW technique was the marginal area. This is in agreement with other studies reported by Stern et al. ${ }^{13}$ and Veljee et al. ${ }^{22}$ It was also noticed that the highest mean gap in the CLW technique group was in the lingual zone which is even greater than the highest mean gap in the SLM technique group (buccal zone) (Tables $3 \mathrm{~A}$ and $\mathrm{B}$ ).

Furthermore, the overall mean gap distance in the CLW technique group was higher than the overall mean of the SLM technique group. However, the difference was not statistically significant $(p>0.05)$.

To conclude, the findings of the present study suggest that RPD frameworks fabricated by the SLM technique generally has a better fit compared to the CLW technique.

Nevertheless, both techniques neither achieved zero gap distance nor had an optimum fit. Many factors could result in a compromised fit of the RPD metal framework. It was observed that one of the most influential factors is the finishing and polishing procedures for the intaglio surface of the framework, particularly the rests. Likewise, it has been reported that controlling the finishing and polishing processes could improve the fit accuracy and that excessive finishing by using stones may result in unnecessary removal of the metal from the internal surface. ${ }^{13}$ Rudd and Morrow ${ }^{23}$ recommended the use of sandblasting and conservative polishing processes to reduce the effect on the intimate fit.

Furthermore, other suggested factors which might affect the final fit of the RPD framework are poor mouth preparation, inaccurate master cast, and defects in its duplication process. ${ }^{24-27}$

Additionally, the cobalt chromium material that is used to produce the complex PRD metal framework is a very important influencer on the final fit accuracy. It was reported that the high-fusing base metal alloys had greaOter solidification shrinkage than gold alloys. ${ }^{28,29}$ Hence, the shrinkage of the cobalt-chromium material during solidification of the metal is expected to compromise the desired fit.

This study had some limitations such as manpower, controlling the finishing and polishing procedures, and handling the PVS specimen as multiple repetitions were required due to its small size and delicacy.

More experimental and clinical trials are needed to evaluate the SLM technique for RPD framework construction. Also, additional studies are needed to compare RPD frameworks fabricated by conventional, milling and 3D printing or SLM techniques. Moreover, comparing different manufacturers or systems for fabrication of RPD framework by computer aid designing and rapid prototyping technology and using intraoral scanning systems are other areas that worth investigations.

\section{Conclusion}

Within the limitations of this study, it can be concluded that RPD metal frameworks fabricated by the SLM technique showed a better fit than the CLW technique. However, this finding was not highly significant. Accordingly, it is expected that the SLM frameworks are cost-effective and with additional in vivo and in vitro clinical studies to support our findings, it can be used to fabricate removable partial dentures.

\section{Clinical significances}

Since the CLW te chnique includes many laboratory procedures which are subjected to accumulative human error, so SLM technique represents an alternative cost-effective method. Selection of either technique is dependent on the dentist preference and the availability of the equipment.

\section{References}

1. Starr JM, Hall R. Predictors and correlates of edentulism in healthy older people. Curr Opin Clin Nutr Metab Care 2010;13(1):19-23.

2. Abt $E, C$ arr $A B$, Worthington $H$ V. Interventions for replacing missing teeth: partially absent dentition. Cochrane Database Syst Rev 2012;(2).

3. Stewart KL, Rudd KD KW. Stewart's Clinical Removable Partial Prosthodontic, 4th edition. 2008.695p.

4. Stefanac S, Nesbit S. Treatment Planning in Dentistry. Treatment Planning in Dentistry. 2007.

5. Van Noort R. The future of dental devices is digital. Dent Mater. The Academy of Dental Materials 2012;28(1):3-12.

6. Rudd RW, Rudd KD. A review of 243 errors possible during the fabrication of a removable partial denture: Part I. J Prosthet Dent 2001;86(3):251-261.

7. Campbell SD, Cooper L, Craddock H, et al. Removable partial dentures: The clinical need for innovation. J Prosthet Dent United States; 2017;118(3):273-280.

8. Kruth J, Mercelis P, Van Vaerenbergh J, et al. Binding mechanisms in selective laser sintering and selective laser melting. Rapid Prototyp J 2005;11(1):26-36.

9. Sun J, Zhang F-Q. The Application of Rapid Prototyping in Prosthodontics. J Prosthodont 2012;21(8):641-644.

10. Yager $\mathrm{S}, \mathrm{Ma} \mathrm{J}, \mathrm{Ozcan} \mathrm{H}$, et al. Mechanical properties and microstructure of removable partial denture clasps manufactured using selective laser melting. Addit Manuf. 2015;8:117-123.

11. Matsumoto $M$, Shiomi M, Osakada K, et al. Finite element analysis of single layer forming on metallic powder bed in rapid prototyping by 
selective laser processing. Int J Mach Tools Manuf 2002;42(1):61-67.

12. Parker MH, Cameron SM, Hughbanks JC, et al. Comparison of occlusal contacts in maximum intercuspation for two impression techniques. J Prosthet Dent 1997;78(3):255-259.

13. Stern MA, Brudvik JS, Frank RP. Clinical evaluation of removable partial denture rest seat adaptation. J Prosthet Dent 1985;53(5):658-662.

14. Azari A, Nikzad S. The evolution of rapid prototyping in dentistry: a review. Rapid Prototyp J 2009;15(3):216-225.

15. Arnold C, Hey J, Schweyen R, et al. Accuracy of CAD-CAM-fabricated removable partial dentures. J Prosthet Dent 2017;1-7.

16. Williams RJ, Bibb R, Eggbeer D, et al. Use of CAD/CAM technology to fabricate a removable partial denture framework. J Prosthet Dent 2006;96(2):96-99.

17. Qian B, Saeidi K, Kvetková L, et al. Defects-tolerant Co-Cr-Mo dental alloys prepared by selective laser melting. Dent Mater. The Academy of Dental Materials 2015;31(12):1435-1444.

18. Kajima Y, Takaichi A, Nakamoto T, et al. Fatigue strength of $\mathrm{Co}-\mathrm{Cr}-\mathrm{Mo}$ alloy clasps prepared by selective laser melting. J Mech Behav Biomed Mater. Elsevier 2016;59:446-458.

19. Almufleh B, Emami E, Alageel $O$, et al. Patient satisfaction with lasersintered removable partial dentures: A crossover pilot clinical trial. J Prosthet Dent 2017;1-9.

20. Akeel R. Effect of edentulousridge length on the fit of occlusal rests of a partial denture metal framework. Dent J 2009;29(2):391-396.
21. Anan MTM, Al-Saadi MH. Fit accuracy of metal partial removable dental prosthesis frameworks fabricated by traditional or light curing modeling material technique: An in vitro study. Saudi Dent J King Saud University; 2015;27(3):149-154.

22. Veljee TM, Shruthi CS, Poojya R. Comparative evaluation of the fit of the partial denture framework fabricated from conventional casting wax and light cured pattern wax- an in vitro study. 2014;2(4): 8-12.

23. Rudd KD. Morrow RM EE. Dental laboratory procedures: Removable partial dentures. J Prosthet Dent 1987;3(5).

24. Dootz ER, Graig RG, Peyton FA. Influence of Investments and Duplicating Procedures on the Accuracy of Partial Denture Castings. J Prosthet Dent. 1965;15:679-690.

25. Lameir BR, Rudd KD SR. Making chromium-cobalt removable patial dentures: a modified technique. J Prosthet Dent 2007;203(11):662 662.

26. Rantanen T. Inaccuracies and defects in frameworks for removable partial dentures. 1986;13:347-353.

27. Brudvik JS, Reimers $D$. The tooth-removable partial denture interface. J Prosthet Dent 1992;68(6):924-927.

28. Earnshaw R. The casting shrinkage of cobalt-chromium alloys. Aust Dent J 1958;3(3):159-170.

29. Anusavice, Kenneth J; Shen, Chiayi; Rawls HR. Phillips' science of dental materials. 2012 